\title{
The problems of hydrocephalus and CSF shunt in the patients with myelomeningocele in their adolescence and adulthood J Yamada*, M Nonaka and M Yamasaki
}

\author{
Address: Department of Neurosurgery, Osaka National Hospital, National Hospital Organization, Osaka, Japan \\ Email: J Yamada* - jyamada@onh.go.jp \\ * Corresponding author
}

from 49th Annual Meeting of the Society for Research into Hydrocephalus and Spina Bifida

Barcelona, Spain, 29 June - 2 July 2005

Published: 30 December 2005

Cerebrospinal Fluid Research 2005, 2(SuppI I):SI6 doi:I0.II86/I743-8454-2-SI-SI6

\section{Background}

Hydrocehalus with myelomeningocele (MMC) is generally considered to be a problem in their early life. Few discussions have been made about the management of hydrocephalus with MMC after adolescence. Furthermore in Japan about 20 years ago closure of myelomeningocele was sometimes performed by orthopedists or pediatric surgeons and very little concerns about hydrocehalus were paid for. Therefore the process of the management of hydrocehaus varies widely among the patients with MMC in the adulthood, some patients are left untreated or longterm malfunctioned or others are intensively followed-up. The aim of this paper is to evaluate the intellectual and social outcome between various management and to report six cases who are underwent VP shunt in the adulthood because of their symptoms due to intemittently increased intracranial pressure are revealed after longstanding so called arrested hydrocephalus.

\section{Materials and methods}

A total of 24(11 male and 13 females) patients with MMC associated hydrocehalus who had been followed over ten years after the initial closure are included in this study

\section{Results}

The patients are classified into three groups. We defined the patients who are intensively followed after the initial CSF shunt and VP shunt are also functioning yet as class1.Class 2 includes the patients whose VP shunt is not functioning and who are considered as a shunt independent and arrested hydrocephalus. The patient in Class 3 has been performed no CSF drainage but show ventricular dilatation. We evaluated intellectual outcome using Wechsler adult intelligence scale(WAIS).

\section{Conclusion}

We conclude hydrocephalus associated MMC is not only the problem in their early life but also the life-long issue. 